\title{
Gestão de Competências e Futuro do Programa NANOSATC-BR, Desenvolvimento de CubeSats, Parceria UFSM - INPE/MCTIC.
}

\author{
Dr. Nelson Jorge Schuch ${ }^{a}$; Eng. Rodrigo Passos Marques ${ }^{a}$; Fernando Sobroza Pedroso ${ }^{\text {a }}$ Eng. Thales Ramos

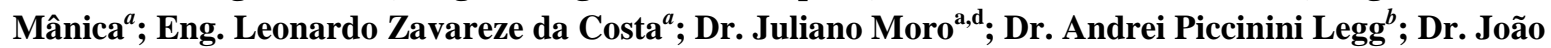 \\ Baptista dos Santos Martins ${ }^{b}$; Dr. André Luís da Silva ${ }^{b}$; Dr. Eduardo Escobar Burger ${ }^{b}$ Dr. Otávio Santos \\ Cupertino Durão ${ }^{c}$; Dra. Fátima Mattiello-Francisco ${ }^{c}$; Dr. Marlos Rockenbach da Silva ${ }^{c}$; Dr. Odim Mendes ${ }^{c}$. \\ [a] Centro Regional Sul de Pesquisas Espaciais - CRCRS/COCRE/INPE-MCTIC \\ [b] Universidade Federal de Santa Maria - UFSM \\ [c] Instituto Nacional de Pesquisas Espaciais - INPE/MCTIC \\ [d] State Key Laboratory of Space Weather, Beijing, China, juliano.moro@inpe.br
}

\begin{abstract}
Resumo: O Programa NANOSATC-BR, Desenvolvimento de CubeSats, trata-se de uma Parceria e Convênio entre a Universidade Federal de Santa Maria - UFSM com o Instituto Nacional de Pesquisas Espaciais - INPE/MCTIC, que teve início em 2006 e atualmente, o Programa conta com dois CubeSats: o NANOSATC-BRI (NCBR1), (1U), lançado em 2014 e ainda em operação, e o NANOSATC-BR2 (NCBR2), (2U), que está em desenvolvimento e tem expectativa de lançamento no primeiro trimestre de 2020. Nesse artigo, é apresentada a Gestão de Competências do Programa, cujo foco, centrado no principal objetivo origem do programa, é a Capacitação Profissional. Destacam-se o envolvimento de alunos de Graduação na concepção, desenvolvimento e operação do NANOSATCBR1, e a participação de alunos de Pós Graduação do INPE no desenvolvimento, verificação e validação do Subsistema de Computador de Bordo para o NANOSATC-BR2. A finalização do desenvolvimento do NANOSATCBR2 e os resultados científicos e tecnológicos do NANOSATC-BR1 são apresentados. O futuro do Programa com o desenvolvimento dos NANOSATC-BR3(NCBR3) e NANOSATC-BR4 (NCBR4) são analisados. São ressaltadas as colaborações com empresas e outras Instituições de Ciência Espacial, Engenharia e Institutos de Tecnologias, Engenharias Espaciais e de Computação Aplicada do Brasil e do Exterior. O Programa recebeu suporte financeiro e tem o apoio da Agência Espacial Brasileira (AEB) e do Ministério da Ciência, Tecnologia, Inovação e Comunicações (MCTIC).
\end{abstract}

Palavras-chave: CubeSats, satélites, capacitação, gestão.

\section{INTRODUÇÃO}

Os nanossatélites do Programa NANOSATC-BR, Desenvolvimento de CubeSats, em execução no âmbito da Parceria e Convênio do Instituto Nacional de Pesquisas Espaciais (INPE-MCTIC), através de sua subunidade, o Centro Regional Sul de Pesquisas Espaciais (CRCRS/COCRE/INPE-MCTIC) com a Universidade Federal de Santa Maria (UFSM) - Convênio MCTIC/INPE-UFSM, e suas Parcerias no Estado: Universidade Federal do Rio Grande do Sul (UFRGS) - Instituto de Informática, Grupo de Microeletrônica UFRGS/II-GME, juntamente com a Santa Maria Design House (SMDH/FATEC-UFSM), utiliza plataforma de satélites do padrão CubeSats dos tipos $1 \mathrm{U}$ e 2U, adquiridas da empresa holandesa Innovative Solutions in Space (ISIS) em Janeiro de 2013, possuindo Modelos de Voo (MV) e Modelos de Engenharia (ME). O NANOSATC-BR1 foi lançado, da Base de Yasny, na Rússia, no dia 19 de Junho de 2014, e segue enviando telemetrias, portanto, há mais de 5 anos. Para o NANOSATC-BR2, segundo Projeto do Programa, as cargas úteis estão (em julho de 2019) sendo integradas e testadas no Laboratório de Simulação e Controle (LABSIM/ETE/INPE-MCTIC), sendo uma delas já operacional (software da AMSAT-Br) e duas em processo de finalização (Sonda de Langmuir e Subsistema de Determinação de Atitude com Tolerância a 
Dr. Nelson Jorge Schuch; Eng. Rodrigo Passos Marques; Fernando Sobroza Pedroso; Eng. Thales Ramos Mânica; Eng. Leonardo Zavareze da Costa; Dr. Juliano Moro; Dr. Andrei Piccinini Legg; Dr. João Baptista dos Santos Martins; Dr. André Luís da Silva; Dr. Eduardo Escobar Burger; Dr. Otávio Santos Cupertino Durãoc; Dra. Fátima Mattiello-Francisco; Dr. Marlos Rockenbach da Silva; Dr. Odim Mendes.

Gestão de Competências e Futuro do Programa NANOSATC-BR, Desenvolvimento de CubeSats, Parceria UFSM - INPE/MCTIC. Falhas) de suas integrações e testes com a plataforma do Modelo de Engenharia (ME). A segunda versão da placa de experimentos do NANOSATC-BR2, agora com componentes de nova geração, foi desenvolvida e fabricada e se encontra em teste finais na SMDH/FATEC-UFSM. O Modelo de Voo (MV) do NANOSATCBR2 está, no momento, na sala 100.000 do Laboratório de Integração e Testes (LIT/INPE-MCTIC), em São José dos Campos-SP, aguardando sua integração final. O lançamento do NANOSATC-BR2 está planejado para ocorrer no primeiro trimestre de 2020. O seu Modelo de Engenharia (ME) encontra-se no LABSIM/ETE/INPE-MCTIC, e está sendo utilizado para os seguintes testes: software de bordo, de solo e integração e comunicação das cargas úteis com a plataforma . Os projetos futuros NANOSATC-BR3 e NANOSATC-BR4 serão desenvolvidos a partir da adaptação dos Modelos de Engenharia dos CubeSats NCBR1 \& NCBR2, com novas cargas úteis e adição dos modelos de voo dos painéis solares. O Programa conta com a participação ativa de alunos de Graduação da UFSM, de alunos das Pós-Graduações do INPE/MCTIC, MD/DCTA-ITA e UFRGS, de Professores da UFSM, UFRGS, Universidade Federal de Minas Gerais (UFMG) e da Universidade Federal do ABC (UFABC), e de Pesquisadores e Tecnologistas do INPE/MCTIC, sendo que alguns deles são ex-alunos egressos da UFSM e do Programa, contratados por meio de concursos públicos pelo INPE/MCTIC e pela própria UFSM, evidenciando a contribuição do Programa para a capacitação de recursos humanos na área. Para mais detalhes sobre o Programa NANOSATC-BR, Desenvolvimento de CubeSats, sugerem-se as leituras de Schuch et al. (2014, 2019). Futuramente, espera-se que os satélites do Programa NANOSATC-BR, Desenvolvimento de CubeSats, possibilitem dispor de uma constelação de nanossatélites Brasileira em órbita espacial.

\section{PROGRAMA NANOSATC-BR}

O Programa NANOSATC-BR, Desenvolvimento de CubeSats é um Programa de caráter pioneiro no Brasil, que visa desenvolver um sistema completo de satélites da classe dos CubeSats, sendo o NANOSATCBR2 o sucessor direto do NANOSATC-BR1. Este último foi lançado, a bordo do lançador ucraniano DNEPR, na Base de Lançamentos de Yasny, na região de Orsk, na Rússia no dia 19 de Junho de 2014. O primeiro nanossatélite do Programa é de padrão $1 \mathrm{U}$, sendo, portanto, um cubo de $10 \mathrm{~cm}$ de aresta, com massa de $0,965 \mathrm{~kg}$. O Modelo de Voo do primeiro CubeSat do Programa, já com os painéis solares montados, pronto para testes, é mostrado na Figura 1.

O NANOSATC-BR1 completou tanto suas missões tecnológicas quanto suas missões científicas e educacionais com sucesso, disponibilizando um vasto banco de dados de housekeeping e de cargas úteis científicas e tecnológicas para os pesquisadores Brasileiros. As operações de rastreio e controle do primeiro satélite do Programa foram realizadas inteiramente por alunos do Projeto na UFSM e ITA, além da operação de outras missões de projetos científicos de satélites nacionais e internacionais. A missão científica do Programa a bordo do NANOSATC-BR1 é coletar dados da intensidade do Campo Magnético Terrestre, em especial na região da Anomalia Magnética da América do Sul (AMAS), que é uma ampla região caracterizada por apresentar baixa intensidade de campo. Além do magnetômetro, para coletar dados do campo magnético da Terra, há dois outros experimentos (ambos bem sucedidos) no NANOSATC-BR1: i) um circuito integrado projetado pela SMDH/FATEC-UFSM e fabricado na Alemanha, com proteção parcial à radiação (metade dele com proteção, e metade sem proteção, para fins de comparação de comportamento entre si) e fruto de demanda da Divisão de Eletrônica Aeropespacial do INPE - DIDEA/ETE/INPE-MCTIC, para possível uso em futuras plataformas nacionais; o Projeto serviu também para validar a biblioteca de software para projetos deste tipo de tecnologia (circuitos com proteção à radiação), antes não existente no Brasil, desenvolvido pela SMDH/FATEC-UFSM, e ii) teste de um software para FPGA, com tolerância a falha para doses de radiação ionizantes instantâneos denominados de Single Event Effects (SEE) e Total Ionization Dose (TID), desenvolvido pela UFRGS/II-GME. 
Figura 1. NANOSATC-BR1 em testes no Laboratório de Integração e Testes - LIT/INPE-MCTIC.

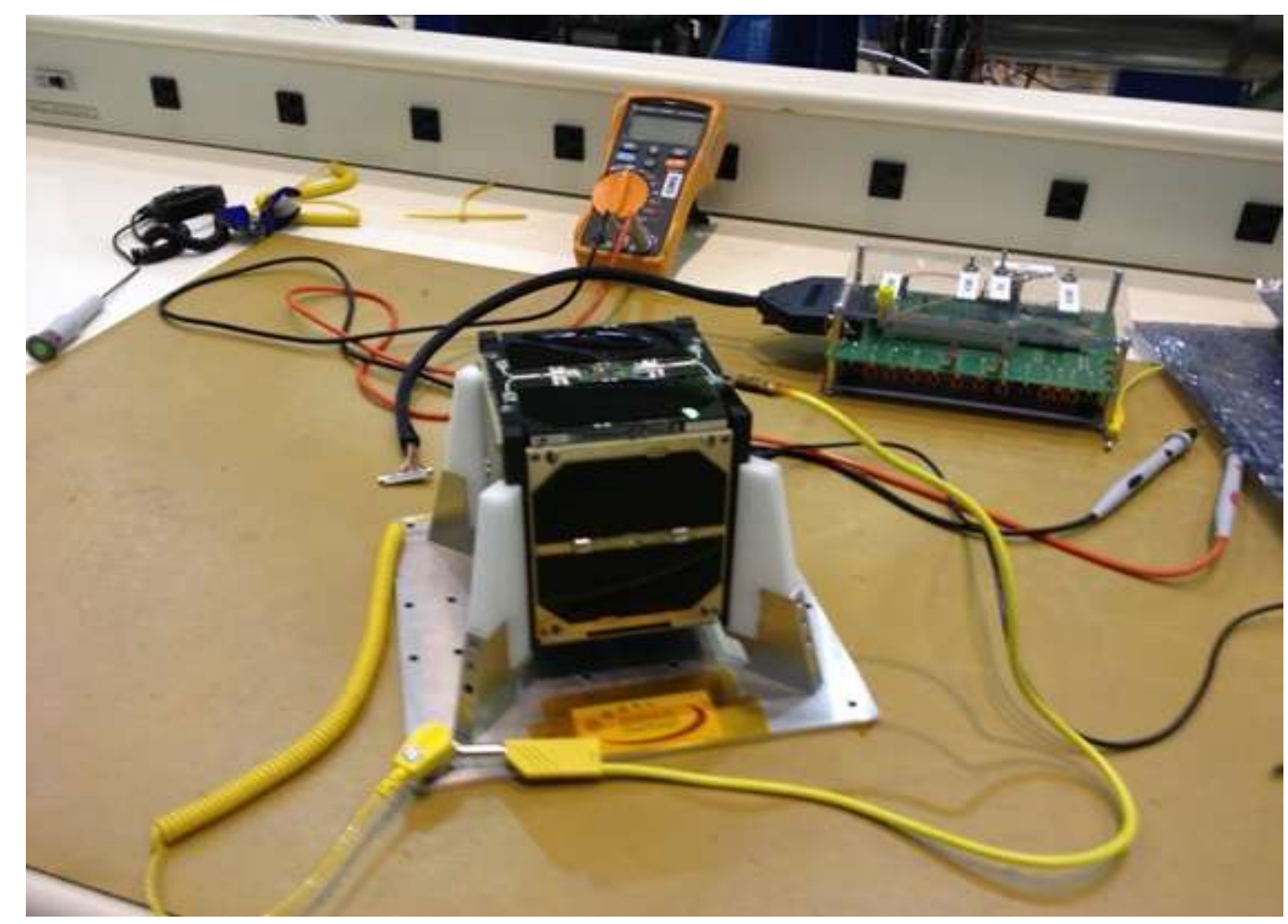

O NANOSATC-BR2 é um nanossatélite do padrão 2U, possuindo os mesmos $10 \mathrm{~cm}$ nas bases, porém a altura é de 22,7 cm (ou seja, 10x10x22,7). As cargas úteis que irão compor a Missão Científica do segundo satélite do Programa NANOSATC-BR são: Sonda de Langmuir, a qual visa coletar dados da Ionosfera Terrestre, e um magnetômetro, que tem o objetivo de realizar a medição da intensidade do Campo Magnético Terrestre com o objetivo de comparação de resultados. Importante ser ressaltado que só há um magnetômetro como carga útil no NANOSATC-BR2. Há dois outros magnetômetros, mas para propósitos diferentes: um no experimento da UFMG, em parceria com o INPE e a UFABC, para uso próprio, e outro na plataforma do NANOSATC-BR2, para determinação de atitude do subsistema de controle do cubesat.

A Missão Tecnológica do NANOSATC-BR2 será validar e testar as seguintes cargas úteis: Experimento de teste do software tolerante a falhas por radiação, que equipará o FPGA ProAsic3, software desenvolvido pela UFRGS/II-GME (este FPGA é diferente e com maior sensibilidade e área de exposição à radiação do que o usado na carga útil do NANOSATC-BR1, com o mesmo propósito); um Circuito Integrado projetado pela SMDH/FATEC-UFSM; um Sistema de Determinação de Atitude com Tripla Redundância Tolerante a Falhas, experimento da UFMG-INPE-UFABC. Há também um sexto experimento no NANOSATC-BR2, que é um software para envio de mensagens entre radioamadores do tipo store-forward, desenvolvido pela AMSAT-Br. Assim sendo, infere-se que se trata de uma Missão-Projeto capaz de envolver várias Instituições de Ensino e Pesquisa Brasileiras, além de empresas com interesse no setor aeroespacial.

\subsection{NANOSATC-BR1: Resultados Científicos}

As medidas do módulo do Campo Magnético Terrestre na região da AMAS, efetuadas com o magnetômetro de três eixos XEN-1210, com resolução de 15 nT, são apresentadas na Figura 2. 
Dr. Nelson Jorge Schuch; Eng. Rodrigo Passos Marques; Fernando Sobroza Pedroso; Eng. Thales Ramos Mânica; Eng. Leonardo Zavareze da Costa; Dr. Juliano Moro; Dr. Andrei Piccinini Legg; Dr. João Baptista dos Santos Martins; Dr. André Luís da Silva; Dr. Eduardo Escobar Burger; Dr. Otávio Santos Cupertino Durãoc; Dra. Fátima Mattiello-Francisco; Dr. Marlos Rockenbach da Silva; Dr. Odim Mendes.

Gestão de Competências e Futuro do Programa NANOSATC-BR, Desenvolvimento de CubeSats, Parceria UFSM - INPE/MCTIC. Figura 2. Medidas do Módulo do Campo Magnético Terrestre na Região da Anomalia da América do Sul, sob o território Brasileiro. Fonte: Schuch et al. (2019).

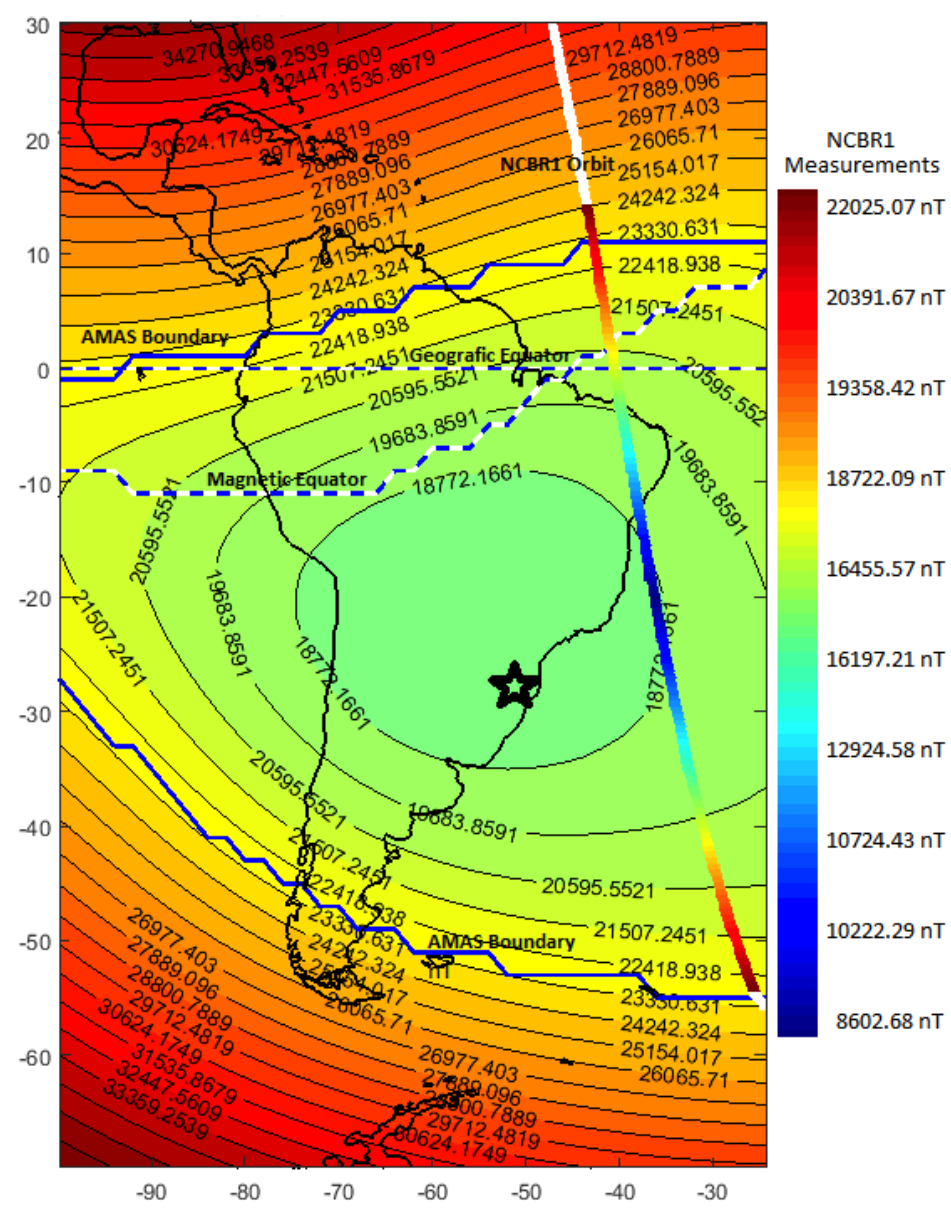

A Figura 2 mostra uma excelente correlação dos dados coletados pelo NANOSATC-BR1 quando comparados com os dados para a intensidade do Campo Geomagnético para a mesma altitude prevista pelo modelo teórico International Geomagnetic Referece Filed (IGRF) para a AMAS.

\subsection{NANOSATC-BR1: Resultados Tecnológicos}

Eventos relacionados ao Clima Espacial, como explosões solares e ejeções de massa coronal também foram observados pelo circuito RH-DRV TestChip-I que foi projetado para resistência à radiação ionizante pela SMDH/FATEC-UFSM, como carga útil tecnológica do NANOSATC-BR1. Um exemplo de dados do fluxo de prótons solares coletados pelo satélite Geostationary Operational Environmental Satellite (GOES15), durante uma tempestade geomagnética que ocorreu entre os dias 10 e 14 de Setembro de 2014, é apresentado na Figura 3.

Na tempestade geomagnética que ocorreu entre os dias 11 e 14 de Setembro de 2014, houve emissões de raios-X, as quais geraram emissões de prótons energéticos solares. O sistema desenvolvido pela SMDH/FATEC-UFSM, o circuito RH-DRVTestChip-I, tolerou a intensa emissão de raios-X na escala R1 e R2. Quando o evento atingiu a escala R3, o circuito RH-DRVTestChip-I revelou alguns erros. A tolerância dos dois registradores do circuito RH-DRVTestChip-I é mostrada na Figura 4. A barra vermelha corresponde à parte do circuito projetado com proteção à radiação pela SMDH/FATEC-UFSM. 
Figura 3. Prótons energéticos solares detectados pelo satélite GOES-15 durante Setembro de 2014. Fonte: Naval et al. (2016).

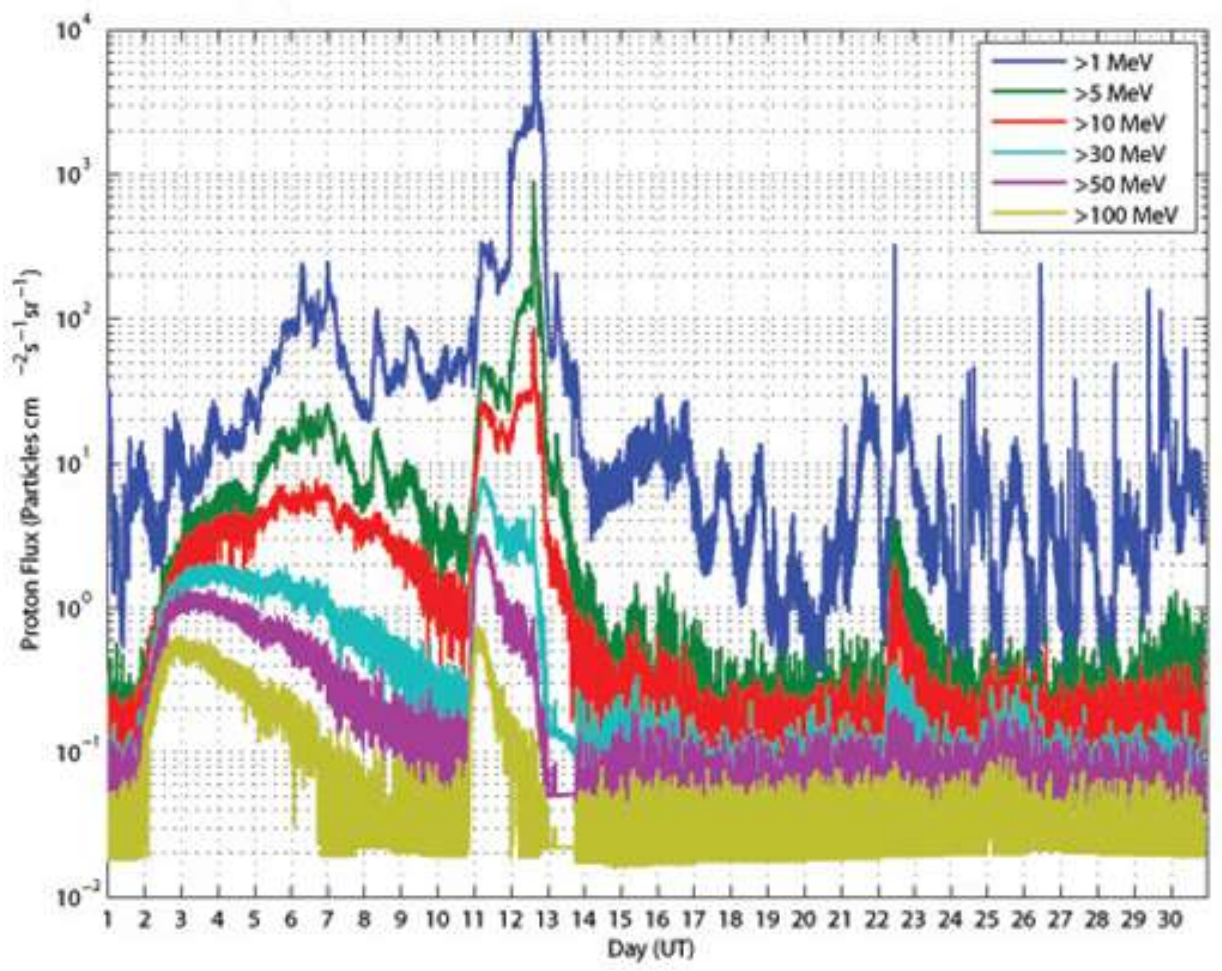

Figura 4. Comparação das tolerâncias do circuito RH-DRVTestChip-I projetado pela SMDH como carga útil tecnológica do NANOSATC-BR1 durante os eventos R1, R2 e R3 - azul parte do circuito sem proteção à radiação. Fonte: Naval et al. (2016).

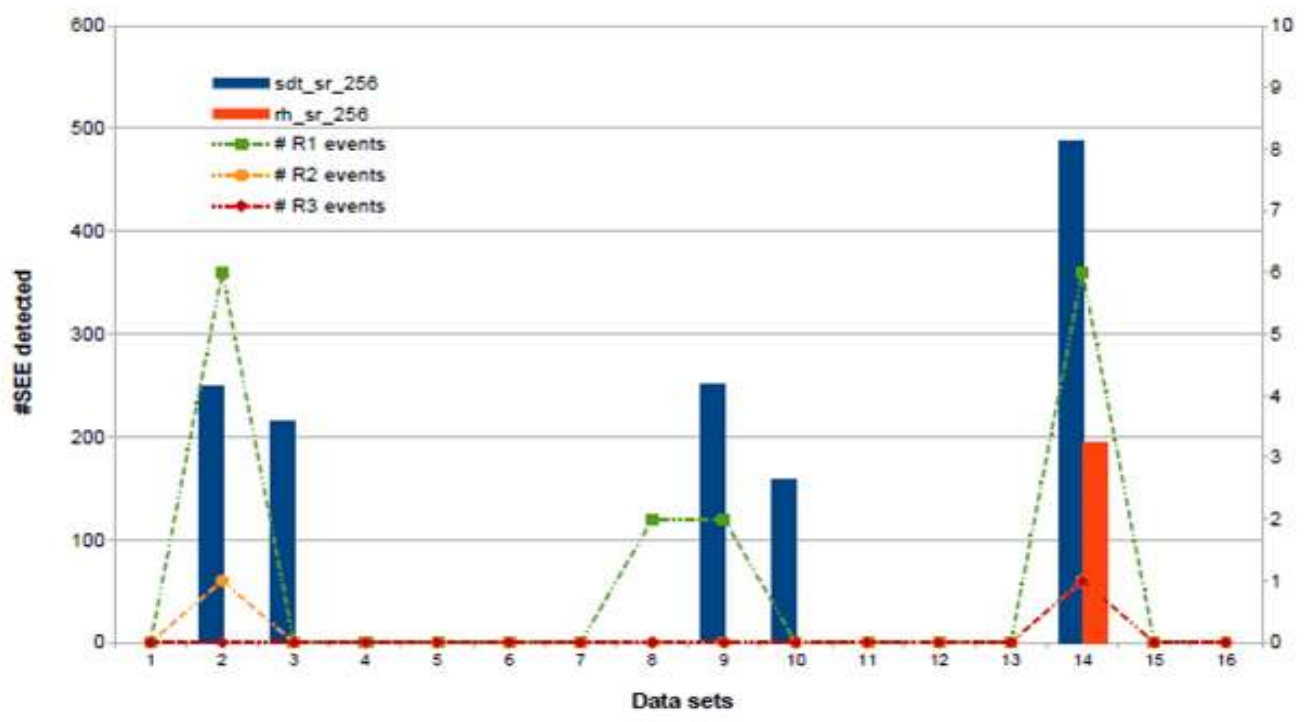

As barras azuis, na Figura 4, de acordo com Naval et al. (2016). correspondem aos registradores de deslocamento projetados usando as células digitais convencionais fornecidas pela fundição. Por sua vez, a barra vermelha representa as células digitais resistentes à radiação projetadas pela SMDH. É notável mencionar que as células projetadas pela SMDH provaram tolerância aos SEE com eventos de gravidade até 
Dr. Nelson Jorge Schuch; Eng. Rodrigo Passos Marques; Fernando Sobroza Pedroso; Eng. Thales Ramos Mânica; Eng. Leonardo Zavareze da Costa; Dr. Juliano Moro; Dr. Andrei Piccinini Legg; Dr. João Baptista dos Santos Martins; Dr. André Luís da Silva; Dr. Eduardo Escobar Burger; Dr. Otávio Santos Cupertino Durãoc; Dra. Fátima Mattiello-Francisco; Dr. Marlos Rockenbach da Silva; Dr. Odim Mendes.

Gestão de Competências e Futuro do Programa NANOSATC-BR, Desenvolvimento de CubeSats, Parceria UFSM - INPE/MCTIC. R1 (linha pontilhada em verde) e R2 (linha pontilhada em amarelo). Em relação ao evento R3 (linha vermelha), as células projetadas relataram alguns erros. A quantidade de erros no registrador de deslocamento projetado usando a biblioteca de células padrão é comparativamente maior que a do registrador de deslocamento usando a biblioteca de células resistentes à radiação, ou "rad-hard". Para a segunda Missão do Programa NANOSATC-BR, os circuitos elétricos tolerantes à radiação foram substancialmente melhorados, e espera-se resultados ainda mais consistentes quanto à resistência à radiação das células digitais em ambiente espacial. A experiência adquirida na primeira Missão do Programa foi o legado fundamental para garantir um retorno de alta qualidade e desenvolvimento tecnológico relevante para a segunda Missão do Programa - NANOSATC-BR2.

\subsection{NANOSATC-BR2: Situação Atual}

Sendo o NANOSATC-BR2 um CubeSat 2U (10x10x22,6 cm), ele permite uma missão mais ambiciosa do que seu antecessor, o NANOSATC-BR1, caracterizando uma planejada e natural progressão tecnológica e com sofisticação da capacidade científica. O Modelo de Engenharia do satélite é representado na Figura 5:

Figura 5. NANOSATC-BR2: Plataforma de serviço e módulo de cargas úteis. Retirado de: http://www.inpe.br/crs/nanosat/centrais_conteudo/galeria/ Acesso em: 28/05/2019.

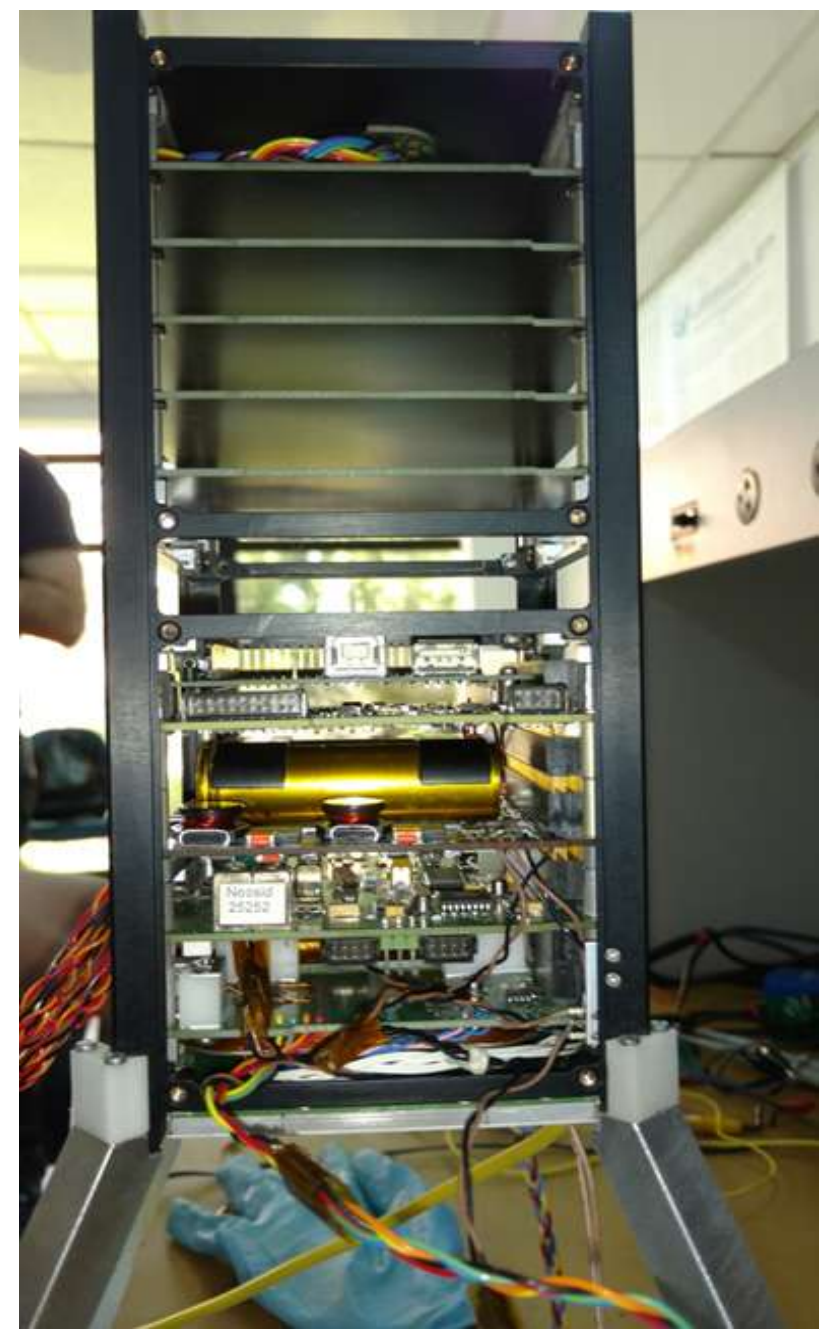

A segunda Missão do Programa possui três objetivos principais: capacitação de recursos humanos, conclusão da missão científica e desenvolvimento de missão tecnológica. A missão científica é monitorar a 
Ionosfera e o Campo Magnético da Terra. Os distúrbios na composição da Ionosfera na região da AMAS, no Brasil, têm graves efeitos sobre as telecomunicações via satélites, bem como, sobre a localização precisa de serviços como o Sistema de Posicionamento Global (GPS). Os equipamentos de carga útil para a missão científica são uma Sonda de Langmuir, e um sensor de campo magnético, XEN-1210, baseado no efeito Hall. A sonda de Langmuir foi miniaturizada para a missão do NCBR2. As suas novas dimensões, em milímetros, são apresentadas no desenho da Figura 6, enquanto que a integração da carga útil à plataforma de serviço é apresentada na figura 7.

Uma vez no espaço, espera-se que o satélite orbite com a Sonda de Langmuir aberta ao lado da estrutura, conforme ilustra a Figura 8.

Para finalizar o NANOSATC-BR2, ainda é necessário (em julho 2019) completar a fase de testes de integração das cargas úteis no modelo de engenharia. O software de gestão de dados a bordo (OBDH) é um elemento-chave para a integração das cargas úteis no NANOSAC-BR2. Trata-se de um software desenvolvido no INPE/MCTIC por alunos de pós-graduação do Curso de Engenharia de Sistemas Espaciais (CSE/INPE-MCTIC), em colaboração com empresas emergentes, também lideradas por ex-alunos do INPE. $\mathrm{O}$ processo de Verificação e Validação (V \& V) está em andamento, abordando as questões de interoperabilidade e robustez nas interações entre o OBDH e as cargas úteis da missão (Conceição et al., 2016) (Batista et al., 2018).

O NANOSATC-BR2 também possui o primeiro subsistema de determinação de atitude de satélites, totalmente desenvolvido no Brasil, como um de seus experimentos, contando com tripla redundância e utilizando três microprocessadores. É um projeto liderado pela UFMG, em parceria com a UFABC e o INPE. O CubeSat NANOSAC-BR2 também levará outros dois experimentos tecnológicos com melhorias em comparação aos que estão voando no NANOSAC-BR1. Ademais, levará um experimento rádio amador da Amateur Radio in Space - Brasil (AMSAT-Br) e da Brazilian Amateur Radio Broadcasting League (LABRE).

Figura 6. Estrutura Interna e Externa da Sonda de Langmuir (dimensões em mm) - ambiente CAD.
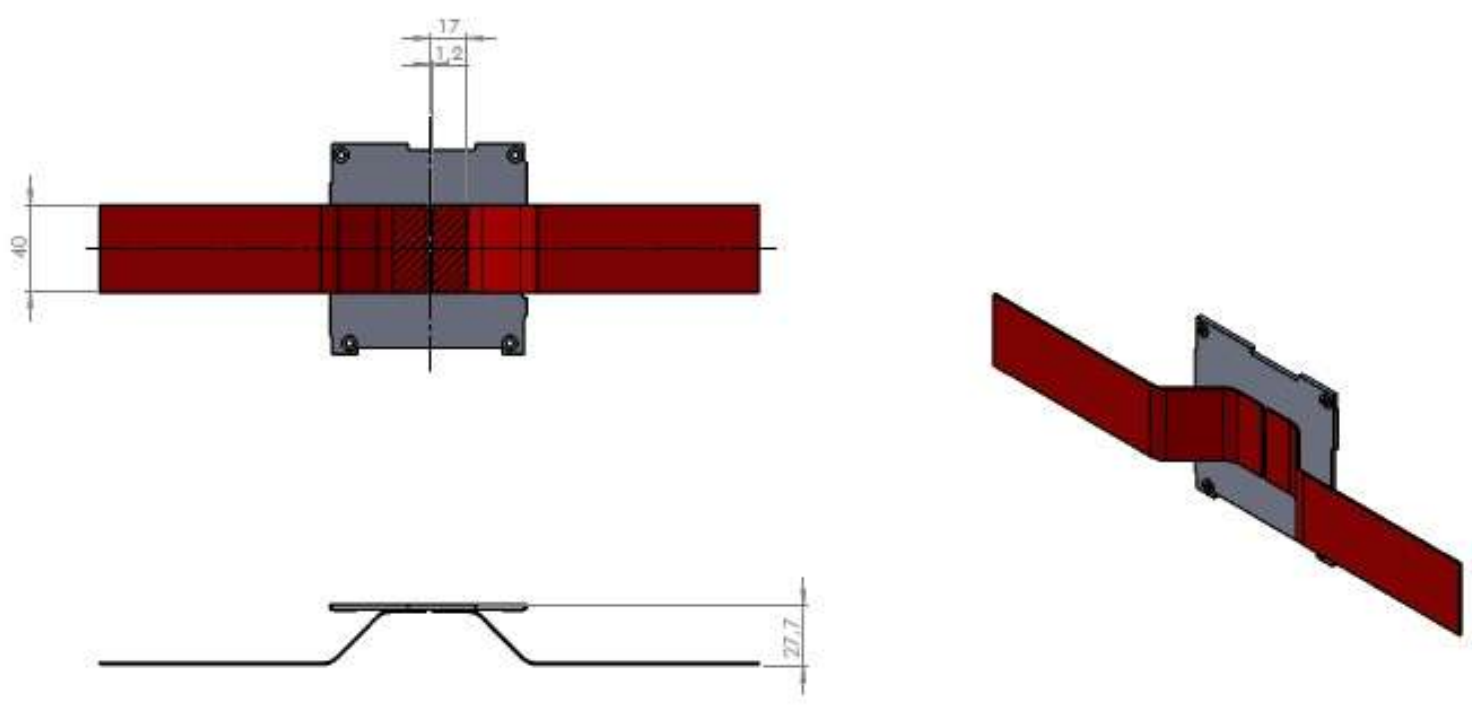
Dr. Nelson Jorge Schuch; Eng. Rodrigo Passos Marques; Fernando Sobroza Pedroso; Eng. Thales Ramos Mânica; Eng. Leonardo Zavareze da Costa; Dr. Juliano Moro; Dr. Andrei Piccinini Legg; Dr. João Baptista dos Santos Martins; Dr. André Luís da Silva; Dr. Eduardo Escobar Burger; Dr. Otávio Santos Cupertino Durãoc; Dra. Fátima Mattiello-Francisco; Dr. Marlos Rockenbach da Silva; Dr. Odim Mendes.

Gestão de Competências e Futuro do Programa NANOSATC-BR, Desenvolvimento de CubeSats, Parceria UFSM - INPE/MCTIC.

Figura 7: Sonda de Langmuir sendo integrada à plataforma de serviço do NANOSATC-BR2.

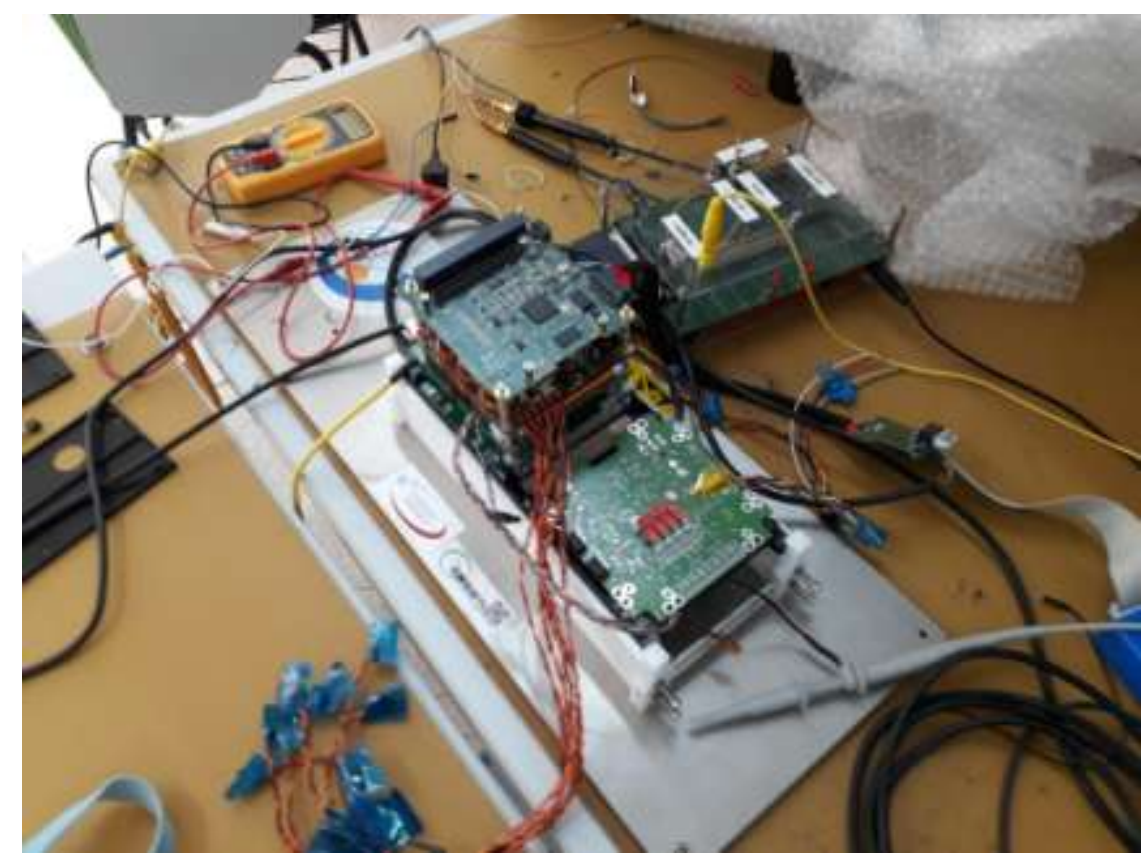

Figura 8. NANOSATC-BR2 em voo - Ambiente CAE (Thermal Desktop).

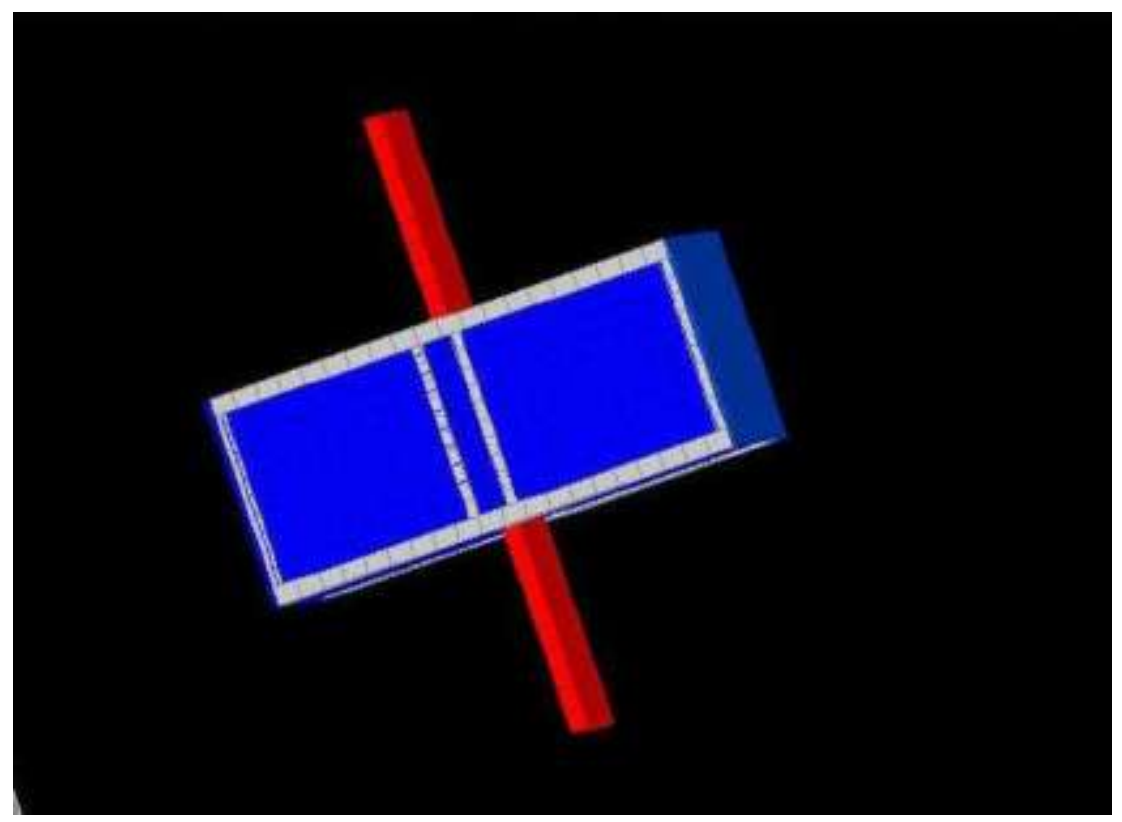

Os experimentos - cargas úteis do NANOSATC-BR2, portanto, são:

- SDATF: Sistema de Determinação de Atitude Tolerante a Falhas - primeiro sistema de determinação de atitude em satélites Brasileiros, com redundância tripla, fabricado nos padrões CubeSat e com algoritmo próprio, utilizando a plataforma do sensor solar e um magnetômetro feito em cooperação pelo INPE/MCTIC com a UFMG (Engenharia Eletrônica) e UFABC (Engenharia Aeroespacial); atualmente, em integração e testes com a plataforma NANOSATC-BR2; 
- Sonda de Langmuir: Entregue para testes e integração com a plataforma NANOSATC-BR2 e o software onboard - OBDH;

- Pacote de Experimentos de Comunicação (store forward) - AMSAT-BR e SP-LABRE.

- Placa CubeSat com três experimentos: FPGA - UFRGS; Magnetômetro; Circuitos tolerantes à radiação - SMDH/FATEC-UFSM.

Todos os experimentos estão sendo integrados e testados (julho 2019) com o software OBDH no Modelo de Engenharia, exceto o experimento elaborado pela SMDH, durante o final da fase de integração.

Todo o software de voo da plataforma foi desenvolvido no Brasil, por pesquisadores e engenheiros do INPE/MCTIC, aproveitando a experiência adquirida no NANOSATC-BR1. O software de controle também foi desenvolvido nacionalmente.

O Programa NANOSATC-BR, Desenvolvimento de CubeSats com os recursos recebidos da Agência Espacial Brasileira (AEB) via UFSM-FATEC contratou a ISIS (julho 2019) para efetuar a gestão de lançamento do NANOSATC-BR2, ficando a operação futura em órbita sob responsabilidade da Equipe de Controle e Rastreio do Programa.- O CubeSat está previsto para ser lançado no primeiro trimestre de 2020. Da mesma forma que o NANOSATC-BR1 e outros projetos CubeSats, o NANOSATC-BR2 será lançado em um lançamento de piggyback, como uma carga terciária, por um veículo de lançamento Soyuz, operado pela Roscosmos, da Rússia.

\subsection{NANOSATC-BR3 \& NANOSATC-BR4: Situação Atual e Planejamento Futuro}

Os modelos de engenharia de ambos os CubeSats NANOSATC-BR1 \& NANOSATC-BR2 estão programados a tornar-se, em breve, os próximos satélites do Programa: NANOSATC-BR3, (NCBR3) \& NANOSATC-BR4, (NCBR4). Portanto, será possível planejar os próximos experimentos científicos e construir novas cargas úteis - experimentos com base na experiência obtida com as missões anteriores.

O Modelo de Engenharia do NANOSATC-BR1 encontra-se atualmente disponível no Laboratório de Integração e Testes para Nanossatélites - LITN/CRCRS/COCRE/INPE-MCTIC, em Santa Maria, RS, conforme ilustra a Figura 9:

Figura 9. Modelo de Engenharia do NANOSATC-BR1 no LITN/CRCRS/COCRE/INPE-MCTIC, em Santa Maria, RS.

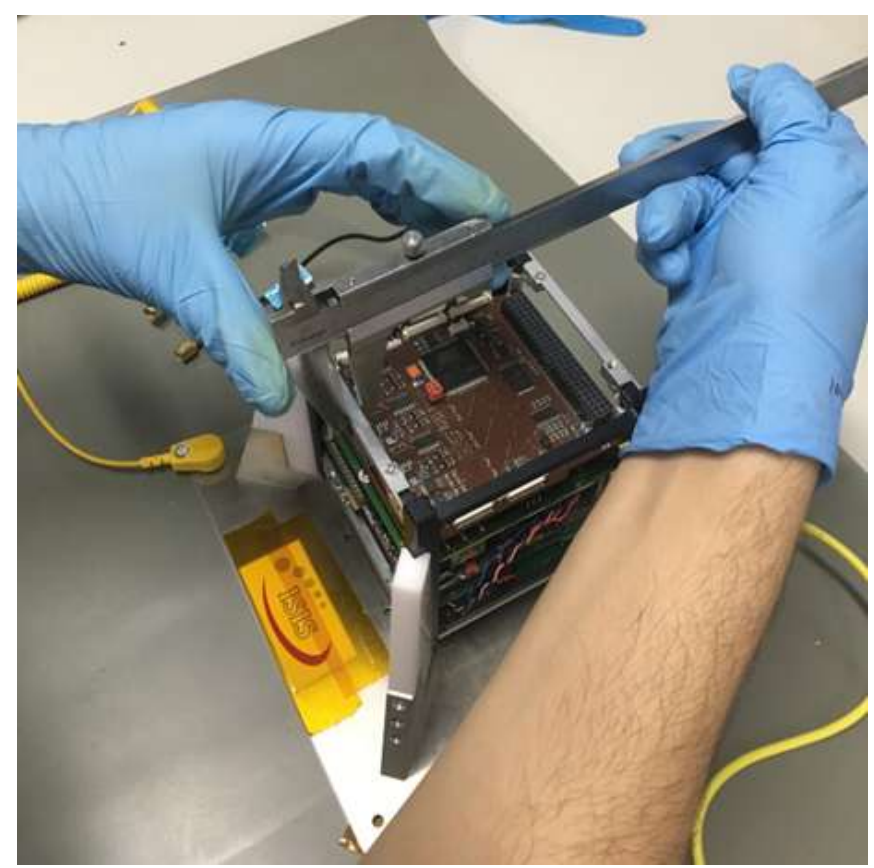


Dr. Nelson Jorge Schuch; Eng. Rodrigo Passos Marques; Fernando Sobroza Pedroso; Eng. Thales Ramos Mânica; Eng. Leonardo Zavareze da Costa; Dr. Juliano Moro; Dr. Andrei Piccinini Legg; Dr. João Baptista dos Santos Martins; Dr. André Luís da Silva; Dr. Eduardo Escobar Burger; Dr. Otávio Santos Cupertino Durãoc; Dra. Fátima Mattiello-Francisco; Dr. Marlos Rockenbach da Silva; Dr. Odim Mendes.

Gestão de Competências e Futuro do Programa NANOSATC-BR, Desenvolvimento de CubeSats, Parceria UFSM - INPE/MCTIC.

A presença do Modelo de Engenharia no Laboratório de Integração e Testes para Nanossatélites LITN/CRCRS/COCRE/INPE-MCTIC, estimula estudantes do recém-criado curso de Engenharia Aeroespacial do Centro de Tecnologia, além de outros cursos relacionados da UFSM, a aprender técnicas de montagem, integração e testes (A I \& T) para CubeSats, além de obter experiência prática com as ferramentas necessárias para a vida diária de um profissional. A fim de efetivamente tornar a plataforma do Modelo de Engenharia em um Modelo de Voo, são necessários diversos ajustes, como a construção de painéis solares, antenas de bordo e o desenvolvimento de placas e componentes de cargas úteis para futuras missões. Todo este processo começará logo após o lançamento do NANOSATC-BR2.

Além do Modelo de Engenharia NANOSATC-BR1, o Modelo de Engenharia do NANOSATC-BR2 será usado para se tornar um dos futuros CubeSats do Programa, o NANOSATC-BR4. Atualmente, o mesmo está sendo testado e utilizado pelos engenheiros de software do INPE/MCTIC, em São José dos Campos SP.

Tanto as futuras cargas úteis quanto as próximas missões científicas serão discutidas com pesquisadores e engenheiros da equipe do INPE/MCTIC, da UFSM e de outras universidades Brasileiras. As futuras missões do Programa NANOSATC-BR representam a continuidade dos Projetos e o início de outros Programas Aeroespaciais no Sul do Brasil para a próxima década.

\section{Gestão de Competências - Capacitação Profissional}

De acordo com informações obtidas em Costa et al. (2010), o Programa NANOSATC-BR, Desenvolvimento de CubeSats, foi implementado em 2006 como uma iniciativa de pesquisa altamente especializada e focada em tecnologia aeroespacial, contando inicialmente com a participação de um aluno do curso de Engenharia Mecânica da UFSM. Ao longo do desenvolvimento do Programa, novos estudantes, oriundos dos diversos cursos de Engenharia e Física da UFSM, envolveram-se com pesquisas na área aeroespacial, dando novas perspectivas e oportunidades de pesquisa, como nas áreas da Física, Mecânica, Elétrica e de Produção (Schuch, et al., 2014).

\subsection{Número de estudantes vinculados ao Programa}

O crescimento do número de estudantes está vinculado ao maior número de bolsas e a maior divulgação do Programa e de seus Projetos.

Atualmente, o principal objetivo do Programa NANOSATC-BR é a Capacitação de Recursos Humanos, por meio da formação de alunos de graduação em suas respectivas áreas, principalmente nas Engenharias: Aeroespacial, Elétrica e Mecânica da UFSM. O número de alunos diretamente envolvidos com o Programa é mostrado na Figura 9. Uma qualidade essencial deste Programa é a participação estreita de estudantes, professores e pesquisadores para o desenvolvimento de atividades focadas em excelência e resultados. 
Figura 9. Número de estudantes vinculados ao Programa NANOSATC-BR: 2006 a 2019.

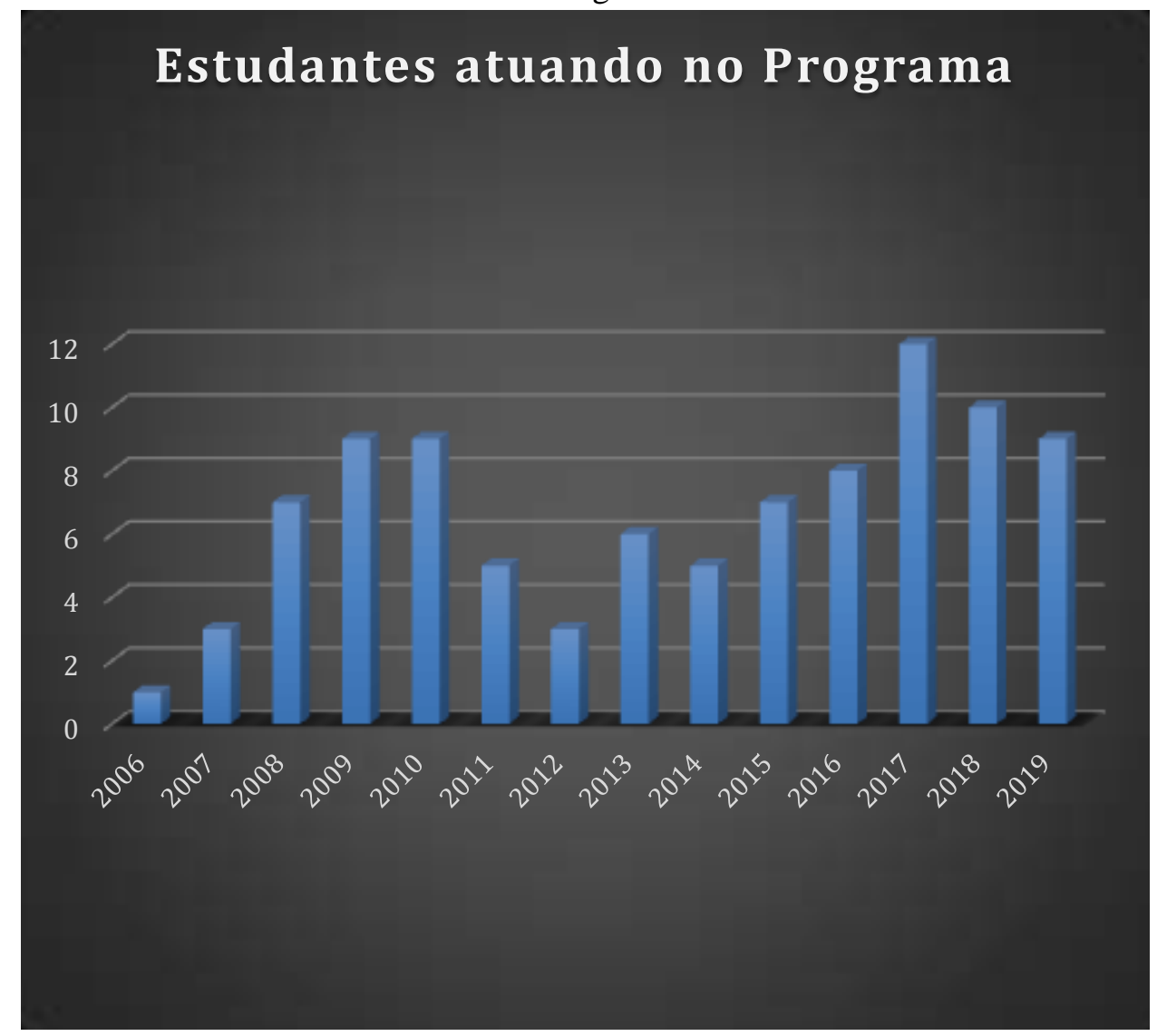

Durante o período de análise do Programa participaram diretamente 34 (trinta e quatro) alunos de nível técnico e nível superior, baseando seus relatórios e Trabalhos Finais de Graduação, com o estudo e conhecimento adquiridos diretamente através do Programa NANOSATC-BR, Desenvolvimento de CubeSats. Existem em andamento dois Trabalhos de Conclusão de Curso - TCC, dos cursos de Engenharia Elétrica e de Engenharia Aeroespacial, que estão em fase final de conclusão, para agosto de 2019, que foram baseados nos dados e projetos de pesquisa do Programa para o desenvolvimento dos seus conteúdos.

Durante o desenvolvimento do Programa contribuíram de forma direta mais de 70 (setenta) pessoas, incluindo pesquisadores e professores de diversas instituições do Brasil.

\subsection{Trabalhos Publicados e Participação em Congressos}

Os trabalhos acadêmicos produzidos pelos alunos englobam boas práticas e técnicas de engenharia, com diversas publicações em congressos e workshops em nível nacional e internacional, todos diretamente relacionados ao Programa, cujas estatísticas são apresentadas no Figura 10. 
Dr. Nelson Jorge Schuch; Eng. Rodrigo Passos Marques; Fernando Sobroza Pedroso; Eng. Thales Ramos Mânica; Eng. Leonardo Zavareze da Costa; Dr. Juliano Moro; Dr. Andrei Piccinini Legg; Dr. João Baptista dos Santos Martins; Dr. André Luís da Silva; Dr. Eduardo Escobar Burger; Dr. Otávio Santos Cupertino Durãoc; Dra. Fátima Mattiello-Francisco; Dr. Marlos Rockenbach da Silva; Dr. Odim Mendes.

Gestão de Competências e Futuro do Programa NANOSATC-BR, Desenvolvimento de CubeSats, Parceria UFSM - INPE/MCTIC.

Figura10. Produção técnica, científica e formação de recursos humanos no período: 2006 a 2019.

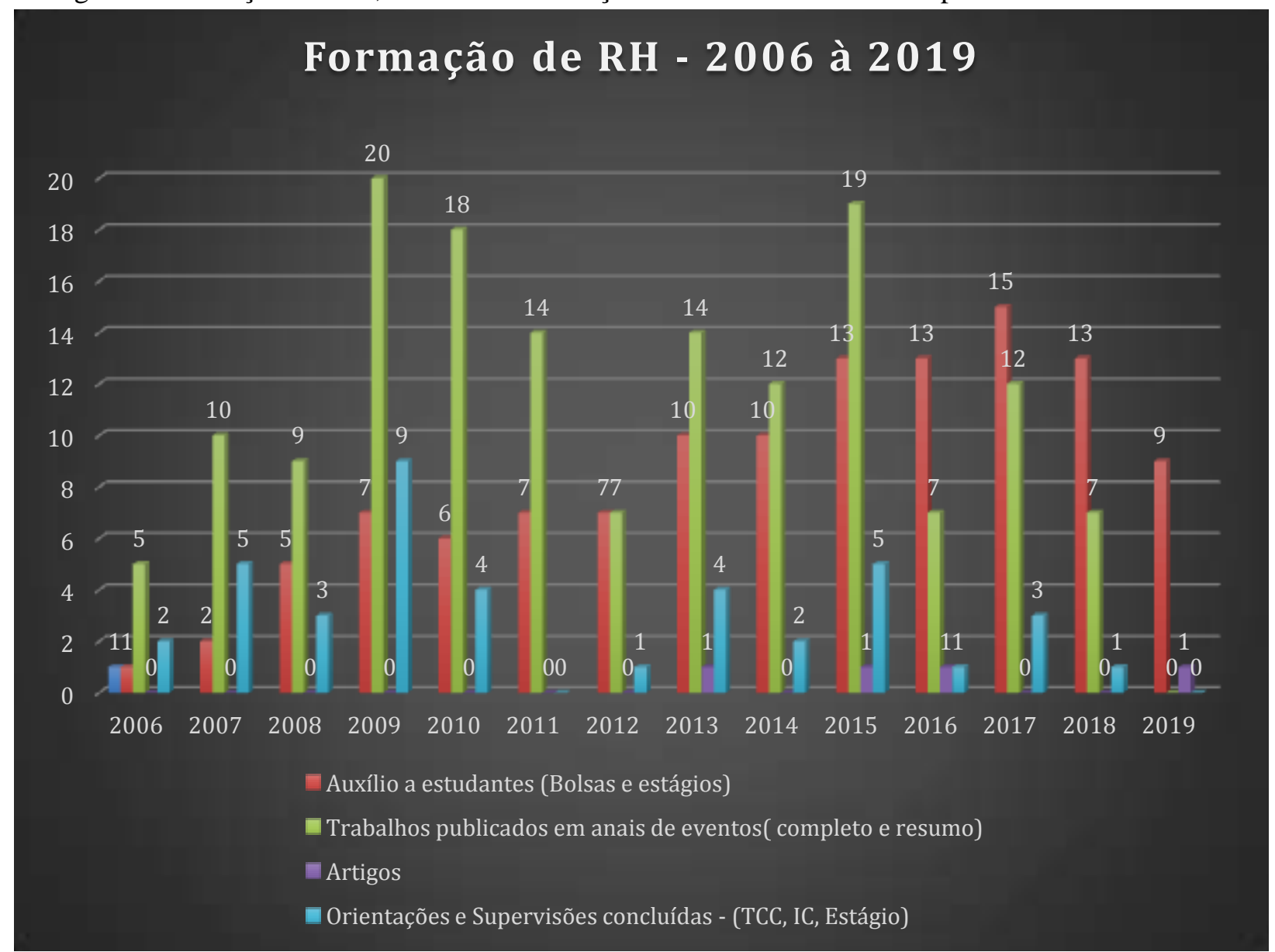

*O número de auxilios foi superior ao número de alunos em alguns períodos devido ao término do vínculo e o início de novo auxilio com o mesmo aluno no ano corrente.

Esta prática de vinculação de alunos no Programa e em seus Projetos realmente melhorou e qualificou o currículo destes alunos e também os motivou para novas iniciativas.

Espera-se que o Programa contribua para agregar tecnologia e capacidade científica às instituições Brasileiras, melhorando sua capacidade em novas áreas tecnológicas e científicas.

Além deste fato, nota-se um aumento no interesse e investimentos do Governo Brasileiro em tecnologia espacial, focando em projetos de nanossatélites, que preenche um nicho crescente e muito próprio, tendo como um exemplo o Programa NANOSATC-BR, Desenvolvimento de CubeSats, Parceria e Convênio UFSM - INPE/MCTIC.

\section{REFERÊNCIAS}

BATISTA, C. L. G.; MARTINS, E.; MATTIELLO-FRANSCISCO, M. F. On the use of a failure emulator mechanism at nanosatellite subsystems integration tests. In: LATIN-AMERICAN TEST SYMPOSIUM (LATS), 19., 2018, São Paulo. Proceedings... IEEE, 2018, p. 1-4.

Disponível em: https://ieeexplore.ieee.org/abstract/document/8347242. Acesso em: 7 mar. 2019.

CONCEIÇÃO, C. L. ; BATISTA, C. L. G.; MARTINS, E.; MATTIELLO-FRANSCISCO, M. F., Dependability verification of nanosatellite embedded software supported by a reusable test system. In: WDES-LADC, 
WORKSHOP ON DEPENDABILITY IN EVOLVING SYSTEMS, CALI, COLOMBIA, BRAZIL, 19 - 21 OCTOBER 2016

COSTA, L. L.; SCHUCH, N. J. ; DURAO, O. S. C. ; BURGER, E. E. ; BOHRER, R. Z. G. . Developing Country's small satellite missions. In: Proceedings of the 61 st International Astronautical Congress - IAC 2010, Prague, Czech Republic.

SCHUCH, N. J. ; ROCKEMBACH DA SILVA, M.; DURAO, O. S. C.. NANOSATC-BR CUBESAT DEVELOPMENT \& CAPACITY BUILDING PROGRAMS: NANOSATC-BR1 \& NANOSATC-BR2, 1st Latin American IAA CubeSat Workshop - IAA-XX-14-0S-0P - dezembro de 2014 - Brasília, Brazil.

SCHUCH, N. J.; DURÃO, O. S. C.; ROCKEMBACK DA SILVA, M.; MATTIELLO-FRANCISCO, F.; DOS S. MARTINS, JOÃO, P. LEGG, ANDREI, L. DA SILVA, ANDRÉ, E E. BÜRGUER, EDUARDO. "THE NANOSATC-BR, CUBESAT DEVELOPMENT PROGRAM - A JOINT CUBESAT PROGRAM DEVELOPED BY UFSM AND INPE/MCTIC - SPACE GEOPHYSICS MISSION PAYLOADS AND FIRST RESULTS" Brazilian Journal of Geophysics [Online], Volume 37 Número 1 (23 março 2019).

NAVAL, J. J. S., MEDEIROS, L., MARTINS, J. B. S., SCHUCH, N. J., DURÃO, O. S. C., MACHADO, R., Design considerations for radiation hardened ASIC used as technological payload in NANOSATCBR1, IAA-BR-16-0S-0P, 2016. 